\title{
Estudio retrospectivo de hallazgos histopatológicos en animales silvestres de vida libre y en cautiverio en Villavicencio, Colombia
}

\author{
A retrospective study of pathological findings in wild and captive- \\ wild animals in Villavicencio, Colombia.
}

\section{Estudo retrospectivo dos achados histopatológicos em animais silvestres de vida livre e cativeiro em Villavicencio, Colômbia}

Yésica M. González- $\mathbf{R}^{1 *}$, Julieta E. Ochoa-Amaya ${ }^{2 *}$, Gustavo González-Paya ${ }^{3 *}$, AdolfK. Ciuoderis-Aponte ${ }^{4}$, Pablo F Cruz-Ochoa ${ }^{* * *}$, Nicolle Queiroz $\mathrm{H}^{6 * *}$, Pablo E. Cruz-Casallas ${ }^{7}$

$1 \mathrm{MVZ} ;{ }^{2} \mathrm{MVZ}, \mathrm{MSc} ;{ }^{3} \mathrm{MV} ;{ }^{4} \mathrm{MVZ} ;{ }^{5 * *} \mathrm{MV} ;{ }^{6 * *} \mathrm{MV}, \mathrm{MSc}, \mathrm{PhD} ;{ }^{7} \mathrm{MVZ}, \mathrm{MSc}, \mathrm{PhD}$.

* Escuela de Ciencias Animales, Facultad de Ciencias Agropecuarias y Recursos Naturales, Programa de Medicina Veterinaria y Zootecnia, Universidad de los Llanos, Villavicencio, Colombia; ${ }^{4}$ University of Wisconsin-Madison, USA;

** Departamento de Patología da Faculdade de Medicina Veterinária da Universidade de São Paulo, São Paulo, SP, Brasil;

7 Instituto de Acuicultura, Facultad de Ciencias Agropecuarias y Recursos Naturales, Universidad de los Llanos, Villavicencio, Colombia.

Email: julietaeochoa@yahoo.es

Recibido: diciembre 15 de 2014. Aceptado: mayo 19 de 2015.

\begin{abstract}
Resumen
El presente trabajo constituye un primer estudio retrospectivo sobre los hallazgos histopatológicos observados en animales silvestres, atendidos en el Laboratorio de Histopatología de la Escuela de Medicina Veterinaria de la Universidad de los Llanos, Villavicencio (Colombia), entre 2004 y 2011. Fueron analizados 43 casos, que incluyeron la presencia de infestaciones parasitarias, neoplasias y reacciones necróticas e inflamatorias. Sesenta y dos por ciento $(\mathrm{n}=31)$ de los animales provenían de vida libre y $28 \%(n=12)$ de cautiverio. La mayoría de los animales atendidos pertenecían a la clase mamíferos, los cuales registraron la mayor frecuencia anual $($ Chi-2, $\mathrm{P}<0.01)$. Las enfermedades infecciosas fueron las más frecuentes, seguidas de las enfermedades no infecciosas y de origen traumático. En reptiles y aves prevalecieron las neoplasias $(\mathrm{Chi}-2, \mathrm{P}<0.01)$. Se observó también que las enfermedades gastrointestinales fueron los hallazgos patológicos más comunes (27\%), seguidos por lesiones pulmonares o respiratorias (18.9\%). El sistema digestivo fue prioritariamente afectado por patologías de origen infeccioso, así como el respiratorio y cardiovascular, mientras que la piel fue afectada por patologías neoplásicas y no infecciosas y el sistema urinario por patologías neoplásicas $($ Chi-2, $\mathrm{P}<0.05)$. Condiciones patológicas del sistema nervioso central fueron las menos encontradas (10.8\%). Entre las condiciones no infecciosas, las más comúnmente encontradas fueron las intoxicaciones y la mayoría de las neoplasias tuvieron origen mesenquimal.
\end{abstract}

Palabras clave: animales silvestres, enfermedades infecciosas, histopatología, patología veterinaria.

\section{Resumo}

O presente trabalho constitui um primeiro estudo retrospectivo dos achados histopatológicos em animais silvestres atendidos no Laboratório de Histopatologia da Escola de Medicina Veterinária da Universidade dos Llanos, Villavicencio (Co- 
lômbia), entre 2004 e 2011. Foram analisados 43 casos, dos quais constaram infecções parasitárias, doenças malignas e reações necróticas e inflamatórias. Sessenta e dois por cento $(n=31)$ eram animais de vida livre e $28 \%(n=12)$ de cativeiro. A maioria desses animais pertencia à classe dos mamíferos, que inclusive registrou a maior taxa anual $(\mathrm{Chi}-2, \mathrm{P}<0.01)$. As doenças infecciosas foram as mais frequentes, seguidas das doenças não infecciosas e traumáticas. Em répteis e aves prevaleceram neoplasias $(\mathrm{Chi}-2, \mathrm{P}<0.01)$. Notou-se também que as doenças gastrointestinais foram achados patológicos mais comuns $(27 \%)$, seguidas por lesões no pulmão ou respiratórias (18.9\%). O sistema digestivo foi principalmente afetado por doenças de origem infecciosa, bem como os sistemas respiratório e cardiovascular, ao passo que a pele foi afetada por doenças neoplásicas e não infecciosas e o sistema urinário por doenças neoplásicas (Chi-2, $\mathrm{P}<0.05)$. Condições patológicas do sistema nervoso central e neoplásicas foram mais raras (10.8 e 8.0\%, respectivamente). Entre as condições não infecciosas, as mais comumente encontradas foram envenenamento e neoplasias mesenquimais.

Palavras chave: animais silvestres, doenças infecciosas, histopatologia, Patologia veterinária.

\begin{abstract}
The present work is a first retrospective study of histopathological findings in wild animals samples received at the Histopathology Laboratory of the School of Veterinary Medicine, University of the Llanos, Villavicencio (Colombia), between 2004 and 2011. 43 cases were analyzed, mainly consisting of parasitic infections, neoplasia and necrotic and inflammatory reactions. Sixty-two percent $(n=31)$ were free life animals and $28 \%(n=12)$ were from captivity. Most of these animals were mammals, who presented the highest annual rate $($ Chi-2, $\mathrm{P}<0.01)$. Infectious diseases were the most frequent, followed by non-infectious and traumatic diseases. In reptiles and birds prevailed neoplasms (Chi-2, $\mathrm{P}<0.01)$. It was also noted that gastrointestinal disorders were the most common pathological findings (27\%), followed by lesions in the lung or respiratory $(18.9 \%)$. The digestive system was mainly affected by diseases of infectious origin, as well as the respiratory and cardiovascular systems, whereas the skin was affected by neoplastic diseases and non-infectious and neoplastic diseases were found in the urinary system $(\mathrm{Chi}-2, \mathrm{P}<0.05)$. Pathological conditions of the central nervous system and neoplasms were rare (10.8 and $8.0 \%$, respectively). Among the non-infectious conditions, the most commonly found were poisoning and mesenchymal neoplasms.
\end{abstract}

Key words: histopathology, infectious diseases, Veterinary pathology, wild animals.

\section{Introducción}

Colombia hace parte de la lista de los 20 países con mayor índice de biodiversidad del planeta, albergando más del 70\% de las especies animales y vegetales conocidas. Por su parte, la Orinoquia Colombiana, por su complejidad orográfica, heterogeneidad de relieve y variedad de climas y microclimas, es una de las regiones con mayor riqueza biológica y, por lo tanto, una de las más biodiversas del país (Garavito - Fonseca et al., 2009).

Una de las consecuencias de los numerosos cambios en los patrones humanos, sociales y ambientales, es la circulación de agentes patógenos que cruzan la barrera de las especies, lo cual afecta tanto a animales silvestres como domésticos, por lo que la comunidad internacional en su conjunto tiene que vigilar, prevenir y controlar las enfermedades de la fauna silvestre, factor esencial para la protección de la salud de los animales y de la humanidad y para las producciones agrícolas y comerciales conexas (OIE 2007).

En Colombia han sido realizados varios estudios retrospectivos sobre patologías en animales silvestres, incluyendo reptiles (Martínez, 2009), aves silvestres de vida libre y cautivas (Soler, 2009) y primates no humanos en cautiverio (Barragán, 1978). Además, la mayoría de los Centros de Diagnóstico analizan y atienden casos patológicos de especies silvestres de forma rutinaria, sobre todo en zonas de alta biodiversidad como los Llanos Orientales; sin embargo, pocas veces estos hallazgos son documentados y socializados, lo cual limita el acceso al conocimiento de las enfermedades que afectan comúnmente a estas poblaciones y su condición sanitaria general.

Entre las patologías más comunes afectando a tortugas silvestres se han reportado fibropapilomatosis, la cual se caracteriza por la aparición de múltiples tumores cutáneos de naturaleza fibroepitelial en cuello, conjuntiva ocular, extremidades, cola, regiones axilares e inguinales, caparazón y plastrón (Jacobson et al., 1998). Histológicamente estos tumores cutáneos muestran hiperplasia epidérmica papilar o proliferación hiperplásica de la dermis, mostrándose como lesión inicial una degeneración vacuolar de las células epidérmicas del estrato basal (Jacobson et al., 1998; Jacobson, 2007).

En canguros se ha reportado toxoplasmosis, parasitosis causada por el Toxoplasma gondii que puede afectar tanto al hombre como a animales mamíferos y aves. El ciclo biológico de este parásito es de tipo heteroxeno, siendo los hospedadores definitivos los felinos, tanto domésticos como silvestres (Moré et al., 2010). En los 
macrópodos se observa como lesiones más importantes congestión pulmonar, edema y consolidación del pulmón. Otras lesiones microscópicas incluyen hemorragias en el miocardio, focos pálidos o malacia cerebral. También se puede encontrar esplenomegalia, linfadenomegalia y a nivel gastrointestinal ulceras. Microscópicamente, la necrosis es la principal lesión, especialmente en el sistema nervioso central, pulmones, nódulos linfáticos, hígado y músculo, acompañada de signos inflamatorios de diverso grado (Ochoa-Amaya et al., 2012).

En aves se ha reportado el tumor de Wilms, una neoplasia maligna trifásica embrionaria derivada de células nefrogénicas blastémicas, células epiteliales y mesenquimales. Son tumores renales malignos, ocasionalmente observadas en aves (Saiz et al., 2003), especialmente en periquitos australianos machos (Anaya-Sinhorini, 2008). La tipología histológica del nefroblastoma consiste en elementos epiteliales y mesenquimales que simulan tejido renal embrionario en diversos grados de alteración, encontrándose desde túbulos bien diferenciados hasta masas lobulares con células no diferenciadas sobre un estroma de tejido celular conectivo. Ocasionalmente, las células epiteliales forman quistes que contienen células secretoras de moco o forman agrupaciones de células escamosas, donde el componente neoplásico predominante consiste en tejido sarcomatoso (Saiz et al., 2003).

En chigüiros, la tuberculosis y lesiones piogranulomatosas son las patologías más frecuentes (Ciouderis y Ochoa, 2010). Las micobacterias son bacilos delgados, rectos o ligeramente curvados, que no presentan esporas; sin embargo, son más resistentes al calor, a cambios de $\mathrm{pH}$ y a procesos de desinfección que otras bacterias no formadoras de esporas. Dentro de este género hay que destacar, por su importancia patógena, M. tuberculosis y M. africanum que causan la tuberculosis humana, $M$. avium, que infecta principalmente a aves, M. microti a roedores y $M$. bovis, cuyos hospedadores son fundamentalmente rumiantes (bovinos, cérvidos, cabra y otros) (Atance y Vizcaino, 1998).

Los granulomas son masas nodulares que histológicamente presentan una zona central de necrosis con neutrófilos, rodeada por histiocitos, macrófagos epitelioides, células gigantes, linfocitos y encapsulada por fibroblastos, los cuales son evidenciados al examen macroscópico como nódulos tuberculosos, frecuentemente asociados a infecciones por miembros del complejo Mycobacterium tuberculosis (CMT) y del complejo Mycobacterium avium (Ginn et al., 2007); no obstante, existen lesiones tuberculoides causadas por agentes infecciosos diferentes a las micobacterias, como son algunos miembros del genero Corynebacterium y Nocardia (Skoric et al., 2007).

Skoric et al., (2007) describieron a las lesiones tuberculoides o semejantes a tuberculosis mostrando la formación de nódulos, con una estructura macro y microscópica de granulomas, los que frecuentemente desarrollan inflamación purulenta local, con marcada infiltración de macrófagos y linfocitos, junto a necrosis caseosa central (Ciouderis y Ochoa, 2010).

En primates no humanos, las micotoxicosis son patologías frecuentes. Las aflatoxinas (AF) son un grupo de metabolitos heterocíclicos sintetizados por algunas cepas de Aspergillus flavus y Aspergillus parasiticus (Murcia, 2010). En la aflatoxicosis, aguda resultante de la ingestión de grandes cantidades de aflatoxinas, se produce necrosis hepática severa. La necrosis es primariamente periportal en pavos, patos, pollos, ratas adultas y gatos; mediazonal en conejos y centrolobular en cerdos, bovinos, perros y cobayas (Carlyle et al., 1996; McGavin et al., 2001; Cifuentes et al., 2006). Los casos de aflatoxicosis crónica, que es la forma más común de la enfermedad, resultan de la exposición a dosis bajas de aflatoxinas por un periodo prolongado de tiempo. Los efectos se desarrollan de días a meses, pero pueden ser evidentes patológicamente en una semana (Hussein y Brasel, 2001).

Las parasitosis gastrointestinales son frecuentes en animales silvestres, principalmente en aquellos mantenidos en cautiverio, debido al estrés del mismo y a los cambios en la dieta. Las enteritis parasitarias se convierten en patología frecuente, como en el caso de los chigüiros (Hydrochaeris hydrochaeris) (Orduz-Latorre et al., 2007), en los cuales se identificaron huevos de Ascaroidea, Trichostrongylus, Trichuris y Ooquistes de Eimeria. Los huevos de áscaris encontrados pueden corresponder a infecciones por Toxocora canis o Neoascaris vitolorum, que parasitan caninos y bovinos y que comparten el hábitat con los chigüiros, pudiendo estos contaminarse con los huevos en las fuentes de agua. Trichostrongylus colubriformis puede parasitar tanto bovinos como conejos e igualmente a los chigüiros (Lapage, 1968). Especies de Trichuris parasitan bovinos, caninos (Trichuris vulpis) y roedores (Trichuris muris), siendo posible que los huevos de Trichuris encontrados en los chigüiros correspondan a las especies anteriormente mencionadas (Quiroz, 1989). Los ooquistes de Eimeria solo se pueden clasificar hasta género, resultando difícil determinar si son ooquistes de Eimerias de bovinos (Orduz et al., 2007; Canizales y Guerrero, 2013).

Las enterobacterias también han sido reportadas afectando a reptiles. En un estudio realizado en São Paulo 
(Brasil), sobre la prevalencia de enterobacterias en $\mathrm{BO}$ trops jararaca, a partir de muestras obtenidas directamente del colón de Jararacas adultas y sanas, fueron observados varios géneros de la familia enterobacteriaceae (Citrobacter, Enterobacter, Escherichia, Kluyvera, Morganella, Proteus, Providencia e Salmonella) y un género de bacteria Gram- negativa, morfológicamente semejante (Aeromonas). Los aislados, más frecuentes fueron Salmonella, Citrobacter y Escherichia. Diferentes autores reportan que los microorganismos encontrados como componentes de la microbiota del sistema digestivo, pueden actuar como agentes etiológicos de enfermedades gastrointestinales en los reptiles (Marçal et al., 2008).

Las gastritis pueden estar relacionadas con infecciones por protozoarios (Cryptosporidium sp o Entamoeba sp) los cuales son causa frecuente de gastritis, enteritis, enterocolitis y mortalidad en reptiles. El Cryptosporidium serpentis ocasiona gastritis necroproliferativa difusa y severa con placas fibrinosas (Martínez - Acevedo, 2009).

Los cérvidos salvajes son afectados frecuentemente por neumonías de diverso origen: infeccioso, parasitario y por cuerpo extraño. Las afecciones respiratorias como bronquitis, bronconeumonías y otras formas de neumonías son comunes en la práctica clínica y patología de los cérvidos, siendo más frecuentes que las afecciones de otros órganos. La bronconeumonía es el tipo más común de neumonía, siendo generalmente causada por bacterias y micoplasmas o por bronco aspiración de alimento y contenido gástrico (BarbantiDuarte, 2007).

La Osteocondromatosis (OC) de los perros y gatos es una anormalidad proliferativa del tejido cartilaginoso que ocurre en los huesos de origen endocondral (Magnussen, 1997; Sarkis et al., 2009; López et al., 2002), caracterizada por el desarrollo de una estructura cartilaginosa encapsulada en la superficie de los huesos (Arruda et al., 2004). En cuanto al origen, las causas de su aparición son algo especulativas (López et al., 2002). En felinos se presenta en edad adulta (Schrader y Sherding, 1994), su desarrollo es progresivo (Magnussen, 1997) y puede ser asintomática hasta que produce compresión de estructuras adyacentes (Arruda et al., 2004) o interfiere en la locomoción (Gee y Doige, 1970).

Finalmente, las microfilariasis son frecuentes en primates no humanos. Las Filarias pertenecen al phylum Nematelmintos y sus larvas se conocen como microfilarias, las cuales alcanzan el espacio sanguíneo o partes del tejido linfático del hospedador. Los huéspedes intermediarios son mosquitos (Culícidos) y pulgas, en los cuales se desarrolla hasta el estadio infectante. En el hospedador final, penetra en tejido conectivo, subcutáneo, sangre, linfa, tendones y cavidades corporales generando obstrucción (Ladino De La Hortúa y Moreno-Orozco, 2007).

En consideración a lo anterior, el objetivo del presente trabajo consistió en clasificar las patologías que afectaron a la fauna silvestre, durante el periodo 2004 2011, atendidas en el Laboratorio de Histopatología de la Universidad de los Llanos.

\section{Materiales y métodos}

\section{Localización del estudio}

Este trabajo se llevó a cabo en el Laboratorio de Histopatología de la Escuela de Ciencias Animales de la Facultad de Ciencias Agropecuarias y Recursos Naturales de la Universidad de los Llanos, localizado a $12 \mathrm{Km}$ del centro urbano del municipio de Villavicencio en el Departamento del Meta, Villavicencio (Colombia).

\section{Descripción metodológica}

Muestras de diferentes tejidos fueron procesadas usando la técnica de rutina de inclusión en parafina y coloración de hematoxilina y eosina para examen histológico (García, 1993). Las muestras fueron individualmente examinadas con magnificación baja y alta y un acercamiento sistemático fue usado para el examen del micro preparado completo.

Inicialmente se realizó un estudio descriptivo, con diagnóstico histopatológico, teniendo en cuenta el diagnóstico preliminar de necropsia, la especie animal, la localización o tejido(s) implicado(s), su etiología y la frecuencia de presentación de la patología.

\section{Análisis estadístico}

Inicialmente los datos fueron analizados mediante estadística descriptiva, para establecer las patologías más frecuentes en cada especie, su etiología y el sistema orgánico más afectado. La incidencia de la enfermedad en cada especie se estimó a partir del número de casos clasificados, divididos por el total de pacientes de cada categoría, atendidos en el Laboratorio de Histopatología durante el periodo de estudio. Para los análisis categóricos de Chi-2 fueron utilizados los softwares SigmaPlot 12.0 y Medcalc 11.5.1.0. Por su parte las figuras fueron realizadas con el programa GraphPad Prism versión 5.0. 
Resultados

La tabla 1 compendia la clase, nombre común, nombre científico, procedencia y el diagnóstico de todas las patologías que afectaron a especies silvestres, de acuerdo con los archivos e historias clínicas del Laboratorio de Patología de la Escuela de Ciencias Animales de la Universidad de los Llanos.

Tabla 1. Clase, nombre común, nombre científico, procedencia y diagnóstico de los casos de patologías que afectaron a especies silvestres, archivados en el Laboratorio de Patología de la Escuela de Ciencias Animales de la Universidad de los Llanos durante el perido comprendio entre 2004 y 2011.

\begin{tabular}{|c|c|c|c|c|}
\hline Clase & Nombre común & Nombre científico & Procedencia & Diagnóstico \\
\hline \multirow{4}{*}{ Reptiles } & Tortuga & Trachemis scripta & Cautiverio & Fibroma \\
\hline & Tortuga & Kinosternon dunni & Cautiverio & Infección TGI \\
\hline & Tortuga & Trachemis scripta venusta & Silvestre & Fibroma \\
\hline & Babilla & Caiman fuscus & Silvestre & Indeterminado \\
\hline \multirow{21}{*}{ Mamíferos } & Ocelote & Leopardus pardalis & Silvestre & Neumonía parasitaria \\
\hline & Mono araña & Ateles fusciceps robustus & Cautiverio & Intoxicación aflatoxinas \\
\hline & Oso melero & Tamandua tetradactyla & Cautiverio & $\begin{array}{l}\text { Infiltración piogranulomatosa } \\
\text { por cuerpo extraño }\end{array}$ \\
\hline & Neque & Dasyprocta puntacta & Silvestre & Enterocolitis mixta \\
\hline & Neque & Dasyprocta puntacta & Silvestre & Neumonía intersticial proliferativa \\
\hline & Chigüiro & Hidrochaeris hidrochaeris & Silvestre & Absceso renal \\
\hline & Chigüiro & Hidrochaeris hidrochaeris & Silvestre & Enteritis mixta, dermatitis superficial \\
\hline & Chigüiro & Hidrochaeris hidrochaeris & Silvestre & Enteritis mixta \\
\hline & Mico & Calicebus cupreus & Silvestre & Enteritis necrótica \\
\hline & Mico nocturno & Aotus lemurinus & SILVESTRE & Necrosis licuefáctica hepática \\
\hline & Oso melero & Tamandua tetradactyla & Silvestre & Hepatitis necrótica \\
\hline & Mico socay & Callicebus cupreus discolor & Cautiverio & $\begin{array}{l}\text { Dilataciones quísticas, y } \\
\text { acumulo de queratina }\end{array}$ \\
\hline & Venado & Mazama americana & Silvestre & $\begin{array}{l}\text { Pleuro-bronconeumonía } \\
\text { crónica activa }\end{array}$ \\
\hline & Ocelote & Leopardus pardalis & Silvestre & Miositis supurativa por trauma \\
\hline & Cuatí & Nasua nasua & Silvestre & $\begin{array}{l}\text { Micocarditis supurativa } \\
\text { crónica activa }\end{array}$ \\
\hline & Armadillo & Dasypus & Cautiverio & $\begin{array}{l}\text { Colitis mixta, gastritis catarral, } \\
\text { endometritis, metritis supurativa. }\end{array}$ \\
\hline & Marsupial & & Cautiverio & Hígado graso \\
\hline & Armadillo & Dasypus & Silvestre & Hiperqueratosis ortoqueratótica \\
\hline & Tigre albino & & Cautiverio & Osteocondromatosis \\
\hline & Chigüiro & Hidrochaeris hidrochaeris & Silvestre & $\begin{array}{l}\text { Reacción piogranulomatosa } \\
\text { supurativa y necrótica pulmonar }\end{array}$ \\
\hline & Oso melero & Tamandua tetradactyla & Silvestre & Gastritis leve \\
\hline
\end{tabular}




\begin{tabular}{|c|c|c|c|c|}
\hline Clase & Nombre común & Nombre científico & Procedencia & Diagnóstico \\
\hline \multirow{15}{*}{ Mamíferos } & Canguro rojo & Macropus rufus & Cautiverio & $\begin{array}{l}\text { Toxoplasmosis sistémica, encefalitis } \\
\text { necrótica asociada a protozoarios. }\end{array}$ \\
\hline & Lapa & Agouti paca & Silvestre & Indeterminado \\
\hline & Chigüiro & Hidrochaeris $h$ & Silvestre & Peritonitis séptica \\
\hline & Armadillo & Dasypus $n$ & Cautiverio & \\
\hline & Lapa & Agouti paca & Silvestre & \\
\hline & Chigüiro & Hidrochaeris hidrochaeris & Cautiverio & $\begin{array}{l}\text { Dermatitis superficial con } \\
\text { hiperqueratosis por ácaros }\end{array}$ \\
\hline & Chigüiro & Hidrochaeris hidrochaeris & Silvestre & Neumonía bacteriana \\
\hline & Chigüiro & Hidrochaeris hidrochaeris & Silvestre & $\begin{array}{l}\text { Enteritis, colitis, dermatitis } \\
\text { asociada a ácaros }\end{array}$ \\
\hline & Armadillo & Dasypus $n$ & Cautiverio & Necrosis hepática \\
\hline & Armadillo & Dasypus $n$ & Cautiverio & Hepatitis y nefritis \\
\hline & Chigüiro & Hidrochaeris $h$ & Cautiverio & Enteritis parasitaria \\
\hline & Nutria & Pteronura brasiliensis & Silvestre & Enterocolitis bacteriana \\
\hline & Mico & Callicebus ornatus & Silvestre & $\begin{array}{l}\text { Neumonía, microfilariasis } \\
\text { en conductillos biliares }\end{array}$ \\
\hline & Venado & Odocoileus virginianu & Silvestre & $\begin{array}{l}\text { Meningoencefalitis, hepatitis, } \\
\text { neumonía supurativa, } \\
\text { peritonitis séptica, metritis. }\end{array}$ \\
\hline & Puerco espín & Coendou sp & Silvestre & Meningoencefalitis zigomicótica \\
\hline Aves & Periquito & Melasicattus ondulatus & Cautiverio & Nefroblastoma \\
\hline
\end{tabular}

Durante el periodo evaluado, la mayor frecuencia de presentación se registró en mamíferos, 38 casos equivalentes al $89 \%$ de la casuística, seguida por reptiles con 4 casos $(9 \%)$ y aves con 1 caso $(2 \%)$ (Figura 1$)$.

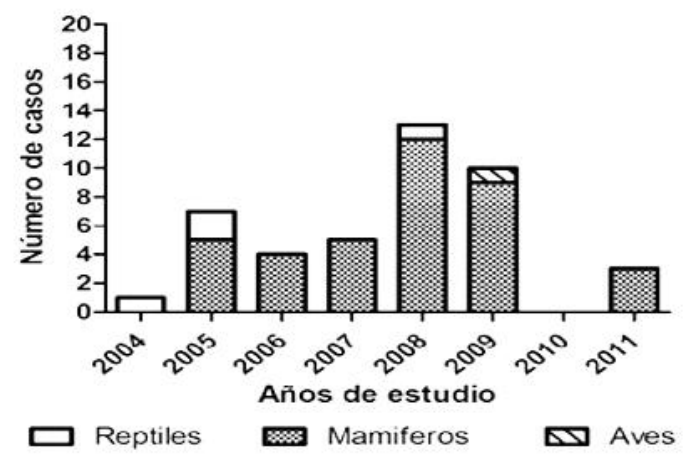

Figura 1. Número anual de casos de patologías de especies silvestres, agrupados por clase de animal afectado, atendidos por el Laboratorio de Histopatologia Veterinaria de la Escuela de Ciencias Animales de la Universidad de los Llanos, en el período comprendido entre 2004 y 2011.
En el análisis por Chi-2 de la frecuencia de animales por año, se observó diferencia significativa $(\mathrm{P}<0.01)$, siendo los mamíferos los que registraron la mayor frecuencia, seguidos de reptiles y aves.

La mayor presentación de enfermedades fueron las de tipo infeccioso con 26 casos $(60 \%)$, las no infecciosas con 12 casos $(28 \%)$ y las neoplasias con 5 casos $(12 \%)$ (Figura 2).

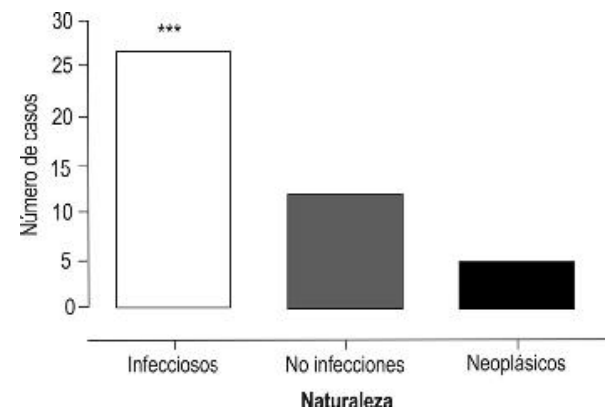

Figura 2. Número de casos de patologías diagnosticados en animales silvestres agrupadas según su naturaleza infecciosa, no infecciosa y neoplásica $\left({ }^{* *}\right.$ Chi-2 $\left.\mathrm{P}<0.01\right)$. 
El análisis de Chi-2 realizado para verificar diferencias en la frecuencia de los tipos de patologías diagnosticadas en relación a las especies, se observó también una mayor frecuencia de patologías infecciosas y no infecciosas en mamíferos, mientras que la mayor frecuencia de patologías neoplásicas fue en reptiles y aves $(\mathrm{P}<0.01)$.

Las enfermedades infecciosas fueron clasificadas por sistemas y el sistema mayormente afectado fue el digestivo con 23 casos (53\%), seguido por el respiratorio con 6 casos $(14 \%)$, reproductor con 4 casos $(9 \%)$, cardiovascular con 3 casos (7\%), nervioso con 3 casos $(7 \%)$, urinario con 2 casos $(5 \%)$ y por último piel y tegumentos con 2 casos (5\%) (Figura 3).

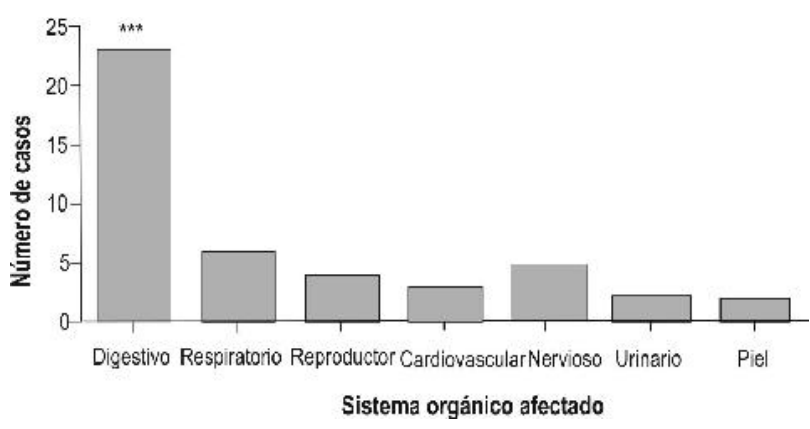

Figura 3. Número de casos de patologías infecciosas agrupadas de acuerdo con el sistema afectado $\left({ }^{* *}\right.$ Chi-2, $\left.\mathrm{P}<0.01\right)$.

Cuando se analizó la relación entre el tipo de patologías por sistema afectado, fueron encontradas mayores frecuencias de patologías neoplásicas y no infecciosas en piel, no infecciosas en tracto digestivo, infecciosas en tracto respiratorio, neoplásicas en tracto urinario e infecciosas en cardiovascular (Chi-2, $\mathrm{P}<0.05)$.

En los casos no infecciosos, el mayor número correspondió a las enfermedades de origen tóxico y traumático - inflamatorias, con 4 casos cada una (37\%), seguidas de las de tipo metabólico con 3 casos $(27 \%)$ y por último las neurogénicas con un caso (9\%) (Figura 4).

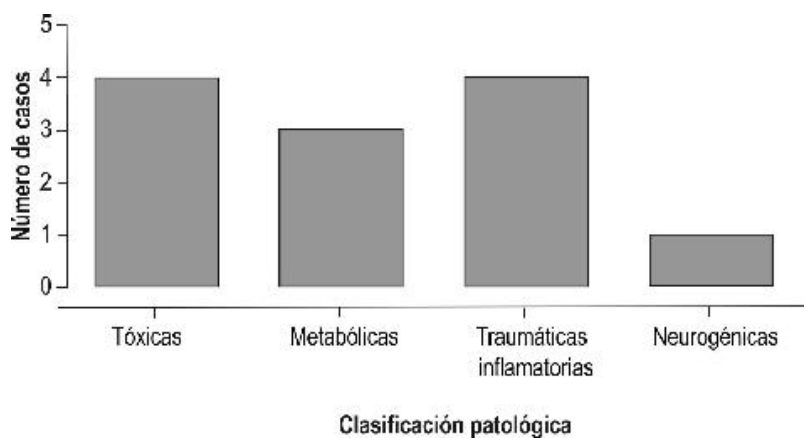

Figura 4. Número de casos de patologías no infecciosas agrupadas de acuerdo con la clasificación patológica
Teniendo en cuenta el origen para la clasificación de las neoplasias, de origen epitelial fue 1 caso $(20 \%)$ diagnosticado, de origen mesenquimal 4 casos $(80 \%)$ y no hubo casos de origen melanocítico $(0 \%)$, para un total de 5 casos (Figura 5).

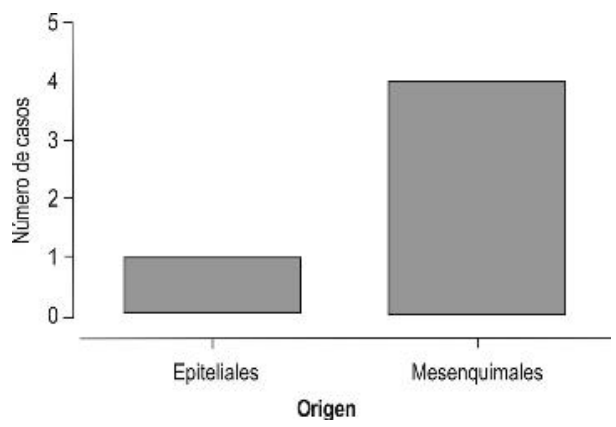

Figura 5. Número de casos de patologías neoplásicas agrupadas de acuerdo al orígen.

\section{Discusión}

En este trabajo, los grupos de animales silvestres más afectados fueron en su orden: mamíferos, reptiles y aves; esto puede explicarse con el hecho de que la Universidad se encuentra en la región de la Orinoquia, la cual cuenta con biodiversidad en fauna silvestre de este tipo, ya que geográficamente tiene ecosistemas para su desarrollo. No obstante estos hallazgos coinciden con lo reportado en un estudio sobre la biodiversidad de la Orinoquia, hecho por la Universidad Nacional, donde resalta que los animales más conocidos y fácilmente observados en los Llanos Orientales incluyen el venado sabanero (Odocoileus virginianus), el chigüiro (Hydróchaeris hydrochaeris) y la corocora roja (Eudocimus ruber) (García et al., 2010).

Además, como se muestra en las figuras 2 y 3, los mamíferos silvestres presentaron mayor casuística de patologías en el periodo comprendido entre los años 2004 y 2011 , con un $89 \%$, seguido de reptiles con $9 \%$ y de las aves con un $2 \%$. Comparadas con las estadísticas presentadas por la Facultad de Medicina Veterinaria y de Zootecnia de la Universidad Nacional de Colombia durante el periodo 2000-2001, de los animales salvajes atendidos, provenientes de diferentes lugares del país, el $50 \%$ de los casos correspondió a aves, el $40 \%$ a mamíferos (principalmente primates) y el $10 \%$ a reptiles. Esto demuestra que en la Universidad de los Llanos se maneja menos casuística de aves, pero en relación con los mamíferos es muy similar en cuanto a los casos en primates no humanos, predominando los casos en el chigüiro, especie endémica de la zona.

En la Figura 4 se puede observar que las patologías no infecciosas de mayor presentación fueron de origen 
tóxico con un $34 \%$, seguido de un $25 \%$ de tipo metabólico, 25\% inflamatorio y $8 \%$ traumático. Estos resultados son similares a los reportados por la Facultad de Medicina Veterinaria y de Zootecnia de la Universidad Nacional de Colombia durante el periodo 2000-2001, donde el $40 \%$ de las aves atendidas fue por trauma y un $15 \%$ por problemas metabólicos; el porcentaje restante correspondía a problemas infecciosos. Con relación a los mamíferos, el $30 \%$ fueron atendidos por problemas metabólicos y un $15 \%$ por traumas, el porcentaje restante igualmente a la aves corresponde a problemas infecciosos y en caso de los reptiles un $45 \%$ correspondió a problemas metabólicos y el 75\% de tipo traumático. En estos datos se observa que es alta la incidencia de traumas, pero se observa que los casos de tipo tóxico son de baja presentación, o no son diagnosticados.

Además, en un estudio retrospectivo realizado por Ortiz et al., (2011), sobre la casuística registrada en el Laboratorio de Patología de la Universidad de Antioquia entre los años 1996 y 2009, se observó que el sistema orgánico más frecuentemente afectado era el reproductivo femenino $(45.6 \%)$ y la reacción más frecuente la de tipo inflamatorio (30.9\%).

En la Figura 5 se observa alta incidencia de las neoplasias de tipo mesenquimal con $80 \%$ y las de tipo epitelial con $20 \%$ de los casos, datos muy similar a los reportados por Zaldivar et al., (2002), quienes muestran que en los caninos se ha observado una amplia presencia de neoplasias de origen epitelial en la piel, en las mamas, intestino y testículo; además, es elevada la incidencia de tumores óseos en esta especie. En los equinos se ha reportado fibrosarcomas y melanomas, carcinoma del pene y del prepucio, de la conjuntiva y de la membrana nictitante. En esta especie no es rara la observación de mielomas y leucemia mieloide (Zaldivar et al., 2002).

En cuanto a las neoplasias en animales silvestres han sido relatadas en todos los sistemas con excepción del SNC. Varias etiologías y factores pueden predisponer a los animales a neoplasias, inclusive virosis. La fibropapilomatosis en tortugas es una enfermedad epizoótica que afecta a las tortugas marinas en todo el mundo, principalmente a la tortuga verde. La etiología aún es desconocida, pero se sospecha de un agente viral. Las lesiones predominantes asociadas con esta enfermedad son fibromas, papilomas cutáneos y fibropapilomas (Hoerner y Baptistotte, 2007, Cubas et al., 2007)

En este estudio, cabe resaltar, que se encontraron dos casos en los años 2004 y 2005, en la especie Trache- mys scripta de fibropapilomatosis cutánea, evidenciando que la casuística disminuyó en los posteriores años debido al poco estudio que se desarrolla sobre esta especie en la Universidad de los Llanos y la tendencia de dirigir la Medicina Veterinaria hacia las especies domesticas exclusivamente, dejando de un lado la biodiversidad de la región. Sin embargo, la proliferación de lesiones cutáneas en las tortugas (Trachemis scripta) las cuales fueron diagnosticadas en los casos del presente trabajo, son similares a los reportados y descritos sobre fibropapilomas de las tortugas verdes en Florida (Jacobson, 2007).

Un estudio realizado en tortugas marinas (Chelonia mydas) del Océano Atlántico en Brasil, muestra la presentación de múltiples papilomatosis cutáneas. Se identificó hiperplasia estromal y de células epiteliales, asociado a cambios que sugerían infección viral, severo polimorfismo y un halo nuclear presentes en los casos de proliferación epitelial y frecuentemente con inclusiones hacia fuera. Todo indicaba que estas lesiones tenían etiología viral, pero fueron negativas para papilomavirus (BPV) y herpesvirus (HSV1 / HSV2) con la técnica peroxidasa-antiperoxidasa (Matushima et al., 2001).

En el presente trabajo se tuvo la oportunidad de diagnosticar un caso de toxoplasmosis en un canguro rojo (Macropus rufus), el cual fue confirmado en el Laboratorio de Enfermedades Emergentes del Departamento de Ciencias Patobiológicas de la Universidad de Winsconsin-Madison (USA) (Ochoa et al., 2012). La infección por toxoplasma ocurre en una amplia gama de animales de sangre caliente, incluyendo a los humanos y es frecuentemente asintomática; sin embargo, puede ser fatal en algunos hospedadores y por ello algunas especies se consideran más susceptibles a la enfermedad como los monos (Casagrande et al., 2013), lémures (Spencer et al., 2004), gatos (Basso et al., 2005) y algunos marsupiales australianos (Basso et al., 2007; Dubey et al., 1988).

En el caso del mico (Callicebus ornatus) que presentó microfilariasis hay que tener en cuenta que en el orden Filaria se encuentran diferentes especies que afectan una amplia gama de hospedadores incluyendo a los humanos y primates no humanos; en estos individuos es más frecuente encontrar microfilarias de forma accidental que el parásito adulto, mostrándose asintomático, pero que pueden causar muerte súbita, como en el caso del presente estudio. Algunos antecedentes como el relatado por Aguilar (1999), en un Colobus (Colobus guereza caudatus), cuyo diagnóstico a la necropsia fue infección espontánea por Dirofilaria immitis (Orihel et al., 1981). 
Ladino (2004) reportó el caso de un Tití gris (Saguinus leucopus) que muere por filariasis cardiaca en el parque zoológico Santa Fe y estudió la prevalencia de microfilaria en 30 primates de dicho zoológico, encontrando microfilarias compatibles con Mansonella ozzardi en sangre de un tití gris (Saguinus leucopus) (Ladino-de La Hortúa y Moreno, 2007).

En primates no humanos las enfermedades gastrointestinales son la causa más frecuente de morbilidad y mortalidad. La diarrea tanto aguda como crónica es la principal manifestación de enfermedad entérica. Al lado de las parasitosis, virosis, dieta y estrés, las bacteriosis constituyen la más importante causa de diarrea. En una colonia de primates del viejo mundo, con más de 1600 ejemplares, el agente bacteriano más frecuentemente aislado en 645 casos de diarrea fue el Campylobacter sp (32\%) seguido por Shigella sp (20\%), Yersinia sp $(10 \%)$, E. coli enteropatogénica (8\%), Aeromonas sp (4\%), Pseudomonas sp (3\%) y Salmonella $s p$ $(1 \%)$, además de las infecciones mixtas (23\%) (Veloso y Catao-Dias, 2007). En este estudio, en un Calicebus cupreus, al que se le diagnosticó por histopatología una enteritis necrótica, no se pudo identificar ningún microorganismo, pues no se realizaron las tinciones especiales, impidiendo la diferenciación de origen bacteriano.

La hepatitis A y B están presentes en algunos chimpancés nacidos en cautiverio y han sido identificadas también en gorilas y orangutanes (Veloso y Catao-Dias, 2007). Las alteraciones necroscópicas incluyen ictericia, hemorragias subcutáneas y musculares, efusiones pleurales y pericárdicas y aumento del hígado, bazo y linfonódulos. Histopatológicamente se observa edema hepatocelular difuso con necrosis y degeneración grasa de hepatocitos, infiltrado mononuclear periportal y cuerpos acidofílicos (corpúsculos apoptóticos de Councilman), que son típicos de la hepatitis de los calitriquídeos (Setzer, 2007).

En una trabajo de maestría cuyo objetivo fue estudiar la frecuencia y la prevalencia de procesos neoplásicos en aves, a través de la Histopatología e Inmunohistoquímica, realizada entre los años 2000 y 2006 en el Hospital Veterinario y en el Departamento de Patología de la Facultad de Medicina Veterinaria y de Zootecnia de la Universidad de Sao Paulo, se observaron 22 tipos tumorales, pero solamente se presentó un caso de nefroblastoma en un periquito australiano (Anaya-Sinhorini, 2008).

Los peces son las presas más importantes de la dieta de las nutrias gigantes; sin embargo, Gómez-Serrano (1999a, 2004) observó que también aves (Aramides cajanea, Eurypyga helias, Jacana jacana, Zenaida auriculata), reptiles (Boa constrictor, Iguana iguana) y mamíferos (Agouti paca, Dasyprocta punctata, Didelphys marsupialis, Philander opossum, Sylvilagus sp., Sciurus $s p$.$) , hacen parte de sus presas. Por su parte, Álvarez-$ León (2008) registró también otros reptiles como babillas, lagartos y tortugas. La nutria gigante estudiada en el presente trabajo, fue diagnosticada con enterocolitis. De acuerdo con su historia clínica su alimentación era a base de peces y serpientes, lo que nos permite inferir que probablemente la enterocolitis fue por el consumo de reptiles que albergan en su microbiota intestinal bacterias Gram negativas y hongos patógenos (Marçal-Bastos et al., 2008).

En el caso de la tortuga (Kinosternon dunni) que presentó una infección gastrointestinal, se puede decir que las enfermedades infecciosas en reptiles son casi siempre el resultado de inmunosupresión, asociada con el estrés post captura. Muchas bacterias Gram-negativas no son consideradas patogénicas en reptiles; sin embargo, algunas bacterias Gram positivas pueden causar enfermedad, especialmente en animales inmunocomprometidos. Diferentes autores reportan que los microorganismos normalmente encontrados como componentes de la microbiota del sistema digestivo pueden actuar como agentes etiológicos de enfermedades gastrointestinales en reptiles (Marçal-Bastos et al., 2008). En estos casos y teniendo en cuenta la presencia de bacterias, Gram (-) se puede asociar a Salmonella spp, ya que es la bacteria más reportada como agente causal de enteritis en reptiles (Jacobson, 2007; Martínez - Acevedo, 2009).

Existen pocos relatos de enfermedades en ñeques (Dasyprocta agutí); sin embargo, en un estudio retrospectivo sobre enfermedades en esta especie, realizado en Brasil, las enfermedades diagnosticadas fueron muerte perinatal por el complejo inanición/ hipotermia $(21,6 \%)$, urolitiasis obstructiva $(6,24 \%)$, distocia $(6,24 \%)$, obstrucción del ciego por arena sablose $(6,24 \%)$, intussuscepción $(3,20 \%)$, fecaloma $(3,20 \%)$ y obstrucción del esófago $(3,20 \%)$ (Batista et al., 2010).

En los casos de Dasyprocta puntacta estudiados fueron diagnosticadas enterocolitis mixta y neumonía intersticial supurativa. Al examen histológico se observó en un segmento del yeyuno necrosis de las criptas del epitelio intestinal, que se extendía a las células de la lámina propia y la submucosa, con una acentuada congestión vascular, hemorragia e infiltrado inflamatorio mixto (células polimorfonucleares y mononucleares) comprometiendo todas las túnicas, lo cual confirma la enterocolitis mixta (Batista et al., 2010). 
Con relación a las dermatitis superficiales con hiperqueratosis diagnosticadas en los chigüiros (Hidrochaeris hidrochaeris), además de estar asociadas con ácaros puede también tratarse de una dermatosis por estrés observada en Dasyprocta sp, Myoprocta sp y en Hidrochaeris hidrochaeris. En esas especies la afección fue identificada en diversas oportunidades en poblaciones de zoológicos, criaderos y áreas verdes urbanas. La patología se caracteriza por lesiones cutáneas en la región dorso lumbar con alopecia y laceraciones de piel. En los casos crónicos con atriquia, fibrosis, hiperqueratinización e hiperpigmentación cutánea extensa. Esas lesiones son secundarias a agresiones intra- específicas por la superpoblación y frecuentemente son confundidas con dermatitis parasitarias (Ribas-Langue y Moreira dos Santos, 2007).

En conclusión, según los archivos del Laboratorio de Histopatología de la Universidad de los Llanos, las patologías que afectan a animales silvestres de la región de los Llanos Orientales son de diverso origen y se reportan en las diferentes especies que son remitidas a este Centro de Diagnóstico.

\section{Agradecimientos}

Este trabajo fue apoyado en parte por una pequeña subvención para la investigación (PRE-03-2010), de la Dirección General de Investigaciones de la Universidad de los Llanos, Colombia.

\section{Referencias}

Aguilar L. 1999. Dirofilaria immitis en un Colobus (Colobus guereza caudatus). American Association of Zoo Veterinarians audobon: 335-336.

Álvarez-León R. Importancia de los peces en la nutrición de la nutria gigante de río (Pteronura Brasiliensis) (carnivora: mustelidae) en Colombia. Revista Luna Azul, 2009;28:8.

Anaya- Sinhorini J. 2008. Neoplasias en aves domésticas y silvestres mantenidas en domicilio: Evaluación Anatomopatológica e Inmunohistoquímica. [Tesis de maestría] Sao Paulo; Universidade de Sao Paulo, Facultad de Medicina Veterinaria y Zootecnia. Programa de Pos- graduación en Patología Experimental y Comparada

Arruda ML, Grevel V, Padilha JG, Abi P, Ludewig E, Canola JC. Hemilaminectomia no tratamento de osteocondromatose vertebral em gato. Ciencia Rural, 2004; 34(6):1953-1956.

Atance PM, Vizcaíno LL. La tuberculosis: Introducción a la enfermedad. Unidad de Enfermedades Infecciosas, Departamento de Patología Animal, Facultad de Veterinaria. Campus Universitario de Espinardo, Universidad de Murcia. Galemys, 1998;10(2):36-46.

Barbanti-Duarte JM. 2007. Artiodactyla - Cervidae (Veado-catingueiro, Veado-campeiro, Cervo do pantanal). En: Cubas Z. S, Silva
J. C. R., Catao-Dias J. L. Editora Roca Ltda. Tratado de Animais Selvagens. Medicina Veterinária. 1st ed. São Paulo, Brasil, p.660.

Basso W, Venturini MC, More G, Quiroga A, Bacigalupe D, Unzaga JM, Larsen A, Laplace R, Venturini L. Toxoplasmosis in captive Bennett's wallabies (Macropus rufogriseus) in Argentina. Veterinary Parasitology. 2007;144(1-2): 157-61.

Basso W, Edelhofer R, Zenker W, Möstl K, Kübber-Heiss A, Prosl $\mathrm{H}$. Toxoplasmosis in Pallas' cats (Otocolobus manul) raised in captivity. Parasitology, 2005;130:293-299.

Barragán - Fonseca K. 1978. Estudio preliminar de especies de micobacterias en primates no humanos en cautiverio en dos centros de rescate de fauna silvestre de Bogotá. Archivo de computador Karol B Barragán F, dir Claudia I Brieva R, dir, asociada Martha I Guerrero G. Bogotá.

Batista JS, Olinda RG, Silva TM, Rodrigues CM, Oliveira AF, Queiroz SA, Morais SR, Oliveira MF. Enfermidades de cutias (Dasyprocta aguti) criadas em cativeiro diagnosticadas pelo exame anatomopatológico. Pesquisa Veterinaria Brasileira. 2010;30(6):497-502.

Canizales I, Guerrero R. Parasitos y Enfermedades parasitarias del Chigüire (Hydrochoerus hydrochaeris). Boletín Académico Ciencias Físicas, Matemáticas y Naturales. 2013;72(1):9-22.

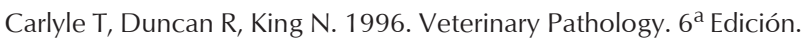
Lippincott Williams \& Wilkins (eds). United States of America. $1392 \mathrm{p}$.

Casagrande RA, da Silva TCE, Pescador CA, Borelli V, Souza Jr JC, Souza ER, Traverso SD. Toxoplasmose em primatas neotropicais: estudo retrospectivo de sete casos. Pesquisa Veterinaria Brasileira, 2013;33(1): 94-98.

Cifuentes CE, Moncaleano JD, Ochoa-Amaya JE. Shock séptico por aflatoxinas en un mono araña (Ateles fusciceps robustus) procedente del bioparque ocarros. Orinoquia, 2006;10(2):15-23.

Ciuoderis-Aponte KA, Ochoa-Amaya JE. Pulmonar pyogranulomatous reaction in wild Capybara (Hydrochaeris hydrochaeris). Orinoquia, 2010;14(1):126-135.

Cubas ZS, Ramos Silva JC, Catão-Dias JL. Tratado de animais selvagens-Medicina veterinária. 1. ed. São Paulo: Editora Roca, 2007. p. 1354

Dubey JP, Crutchley C. Toxoplasmosis in Wallabies (Macropus rufogriseus and Macropus eugenii): blindness, treatment with Atovaquone, and Insolation of Toxoplasma gondii. Journal of Parasitology, 2008;94(4):929-933.

Garavito-Fonseca J, Nino M, Utrera A. 2009. Modelación de hábitat para el ordenamiento territorial ambiental en Casanare-Colombia. En: Ramírez Gil H, Quiñones-Q L, Santana Castañeda E. Libro de resúmenes, Primer Congreso Internacional de Biodiversidad de la Cuenca del Orinoco. Programa de Biología. Instituto de investigaciones de la Orinoquia colombiana. Universidad de los Llanos, p.27.

García R. 1993. Laboratorio de Anatomía Patológica. Interamericana McGraw Hill. España.: 654. 
García J, Defler TR, Bueno ML. The Conservation Status of Callicebus caquetensis (Pitheciidae): A New Species in Southern Caquetá Department, Colombia. Neotropical Primates. 2010;17(2):37-46.

Gee B.R, Doige C.E. Multiple cartilaginous exostosis in a litter of dogs. J Am Vet Med Assoc. 1970;156(1):53-59.

Ginn PE, Mansell JE, Rakich PM. Skin and appendages. En: Jubb, K., Kennedy, P., Palmer, N. Pathology of Domestics animals. Elsevier Saunders. 2007;2(1):687.

Gómez-Serrano JR. 1999a. Ecología alimentaria de la nutria gigante (Pteronura brasiliensis) en el bajo Río Bita Vichada, Colombia. Tesis Profesional. Facultad de Ciencias Pontificia Universidad Javeriana. $50 \mathrm{p}$

Gómez-Serrano JR. 2004. Ecología alimentaria de la nutria gigante (Pteronura brasiliensis) en el bajo Río Bita Vichada, Colombia, pp. 203-224 In: Díaz Granados-Pitti, M. C. \& F. Trujillo-González (eds.) Estudios de fauna silvestre en ecosistemas acuáticos en la Orinoquia colombiana. Fundación Omacha / IIRBAvH / GTZ / Pontificia Univ. Javeriana - IDEADE / DET. Santa Fe de Bogotá D. C. (Colombia), Serie Investigación, 6, 403 p.

Hoerner P, Baptistotte C. Chelonia. 2007. Tartaruga, Cágado, Jabuti. En: Cubas ZS, Silva JCR, Catao-Dias JL. Editora Roca Ltda. Tratado de Animais Selvagens. Medicina veterinária. 1st ed. Sao Paulo, Brasil, p. 109- 110.

Hussein H, Brasel J. Toxicity, metabolism, and impact of micotoxins on humans and animals. Toxicology, 2001;167:101-134.

Jacobson E, Lackovich J, Klein K, Brown S, Homer D, Garber B, Mader R, Moretti D, Patterson R, Herbst AD, Lawrence $H$, Oros J. 1998. Prevalence and cultivation of a Chelonid herpesvirus associated with Fibropapillomas of the green turtle, Chelonia mydas, and the loggerhead turtle, Caretta caretta, in Florida. Gainesville, Florida Cooperative fish and wildlife research Unit university of Florida.

Jacobson E. 2007. Infectious diseases and Pathology of reptiles. 1. ed. Boca Raton, Florida: CRC Press Taylor and Francis Group, $731 \mathrm{p}$.

Jubb-Kennedy, Palmer, N. Pathology of Domestics animals. Elsevier Saunders. 2007;2:608-610.

Ladino-De la Hortúa R, Moreno-Orozco M. Prevalencia de Microfilaria spp en primates de zoológicos colombianos. Revista de Medicina Veterinaria, 2007;13:83-94.

Ladino- De la Hortúa R. 2004. Prevalencia de microfilaria en primates del Zoológico Santa Fe, Trabajo de clínica Ambulatoria de Medicina Veterinaria, Universidad de la Salle, 2004. Entrevista con Marcos Restrepo, Medico humano Instituto Colombiano de Medicina Tropical "Antonio Roldan Betancur". Medellín, 10 de mayo de 2004.

López C, Romina L, Fogel-Nasello F. 2002. Sinovioma maligno: sus diagnósticos diferenciales. Universidad Nacional del Centro de la Provincia de Buenos Aires (Tandil) - Argentina Facultad Ciencias Veterinarias. Área de Clínica Médica y Quirúrgica de Pequeños Animales.

Magnussen KL. What is your diagnosis? Journal of the American Veterinary Medical Association, 1997;210(12):1733-1734.
Marçal-Bastos H, Laranjeira Lopes LF, Gattamorta MA, Matushima E. Prevalence of enterobacteria in Botrops jararacá in São Paulo State: microbiological survey and antimicrobial resistance standarts. Acta Sci. Biol Sci. 2008;30(3):321-326.

Martínez-Acevedo L. 2009. Estudio retrospectivo de la casuística de reptiles en el Laboratorio de Patologia Veterinaria de la Universidad Nacional de Colombia. [Tesis de especialización] Bogotá: Universidad Nacional de Colombia, Facultad de Medicina Veterinaria y Zootecnia. Departamento para la Producción Animal - Departamento de Ciencias para la Salud Animal.

Matushima E, Longatto F, Celso D, Loretto, Kanamura C, Sinhorini I, Gallo B, Baptistolle C. Cutaneous papillomas of green turtles A morphological, ultra-structural and Immunohistochemical study in Brazilian specimens. Vet Res anim Sci. 2001;38(2):51-54.

McGavin D, Carlton W, Zachary J. 2001. Thomson’s Special Veterinary Pathology. $3^{\text {a }}$ edition. Mosby, Inc. An affiliate of Elsevier. Printed in the United States of America.

Moré G, Pardini L, Basso W, Machuca M, Bacigalupe D, Villanueva MC, Schares G, Venturini MC, Venturini L. Toxoplasmosis and genotyping of Toxoplasma gondii in Macropus rufus and Macropus giganteus in Argentina. Veterinary Parasitology, 2010;169(1-2):57-61.

Murcia HW. Micotoxinas y Aflatoxina B1, un problema en salud animal. Revista Teoría y Praxis Investigativa. 2010;5(2):71-8.

OIE. 2007. Enfermedades de la Fauna Silvestre. Organización Mundial de Sanidad Animal \& Oficina Internacional de EpizootiasOIE. [En línea, Acceso 27 Enero de 2009]. URL: http://www. oie.int/esp/ressources/wild_es_final.pdf.

Ochoa JE, Ciuoderis KA, Lim A, Bolin S, Langohr I. Toxoplasmosis sistémica en un canguro rojo (Macropus rufus) cautivo. International Journal of Morphology, 2012;30(1):70-76.

Orduz-Latorre S, Mesa LE, Villar-Cleves C. 2007. Parásitos gastrointestinales en chigüiro (Hydrochaeris hydrochaeris). Asociación Colombiana de Médicos Veterinarios y Zootecnistas. 37(1)/ edición 104

Orihel. Susceptibilidad de primates de laboratorio a la infección con Mansonella ozzardi humana. The American Society of Tropical Medicine and Higiene. 1981;30(4):790-794.

Ortiz L, Garzón A, Rodríguez B. Cytodiagnosis trends at the animal pathology laboratory of the University of Antioquia (Colombia), Revista Colombiana de Ciencias Pecuarias. 2011;24(2):157169.

Ribas-Langue R, Moreira dos Santos E. 2007. Rodentia - Roedores Silvestres (Capibara, Cutia, Paca, Ourico). En: Cubas Z. S, Silva J. C. R., Catao-Dias J. L. Editora Roca Ltda. Tratado de Animais Selvagens. Medicina veterinária. 1st ed. São Paulo, Brasil, p. 481.

Saiz de la Maza R, Vérez-Fraguela JL, , Bonastre C, Cerdeira P. Nefroblastoma Embrionario Canino. 2003. Revista AVEPA. 2003;23(1):45-49.

Sarkis AL, Noyma M, Azevedo E, Lavalle GE, de Lima R. Osteocondromatose em um dachshund: relato de caso. Clínica Veterinária. 2009;79:38-42. 
Schrader S, Sherding R. 1994. Disorders of the skeletal system. In: Sherding R. The cat diseases and clinical management. 2 ed. New York: Academic, v 2: 1599-1647.

Setzer AP. 2007. Hepatites Virais. En: Cubas Z. S, Silva J. C. R., Catão-Dias J. L.Editora Roca Ltda. Tratado de Animais Selvagens. Medicina Veterinária. 1st ed. São Paulo, Brasil, p. 817.

Skoric M, Shitaye EJ, Halouzka R, Fictum P, Trcka I, Heroldova M, Tkadlec E, Pavlik I. Tuberculous and tuberculoid lesions in free living small terrestrial mammals and the risk of infection to humans and animals: a review. Veterinary Medicine. 2007;52(4):144-161

Spencer JA, Joiner KS, Hilton CD, Dubey JP, Toivio-Kinnucan M, Minc JK, Blagburn BL. Disseminated toxoplasmosis in a captive ring-tailed lemur (Lemur catta). Journal Parasitology. 2004;90(4):904-6.
Thompson K. 2007. Tumor and tumors-like lesions of bones. En: Jubb, K \& Palmer's Pathology of Domestic Animals. Elsevier Saunders. 5 ed. Vol I. p 119.

Veloso AL, Catão-Dias J. L. 2007. Primates-Primatas do Velho mundo (Babuíno, Mandril, Chimpanzé, Orangotango). En: Cubas Z. S, Silva J. C. R., Catão-Dias J. L. Editora Roca Ltda. Tratado de Animais Selvagens. Medicina veterinária. 1st ed. São Paulo, Brasil, p 389.

Zaldívar N, Puebla H, Zaldívar Y, Almaguer Y. Importancia de las neoplasias en los animales domésticos. Neoplasias diagnosticadas en los centros de epizootiología y diagnóstico veterinario de la Facultad de Medicina Veterinaria, Universidad de Granma, Cuba. Revista de Producción Animal. 2002;14(1):51-53. 